\title{
Quantitative STEM: Comparative Studies of Composition and Optical Properties of Semiconductor Quantum Structures
}

\author{
Andreas Rosenauer ${ }^{1}$, Florian Fritz Krause ${ }^{1}$, Knut Müller-Caspary ${ }^{1}$, Elias Goldmann², Frank Jahnke ${ }^{2}$, \\ Matthias Paul ${ }^{3}$, Michael Jetter ${ }^{3}$, Peter Michler ${ }^{3}$, Marcus Müller ${ }^{4}$, Peter Veit ${ }^{4}$, Jürgen Christen ${ }^{4}$, Tilman \\ Schimpke ${ }^{5}$, Jan-Philipp Ahl ${ }^{5}$, Adrian Avramescu ${ }^{5}$ and Martin Strassburg ${ }^{5}$. \\ 1. Institute of Solid State Physics, University of Bremen, 28359 Bremen, Germany \\ 2. Institute of Theoretical Physics, University of Bremen, 28359 Bremen, Germany \\ 3. Institut für Halbleiteroptik und Funktionelle Grenzflächen, Universität Stuttgart, 70569 Stuttgart, \\ Germany, \\ 4. Institute of Experimental Physics, Otto-von-Guericke-University Magdeburg, 39106 Magdeburg, \\ Germany \\ 5. OSRAM Opto Semiconductors GmbH, 93055 Regensburg, Germany
}

Optical properties of semiconductor quantum structures are governed by their size and composition. Understanding and interpreting optical measurements require structural data that can be obtained by transmission electron microscopy (TEM). This will be demonstrated in this study for different examples of ternary [1,2] and quaternary [3,4] semiconductor quantum well (QW) and quantum dot (QD) samples by Z-contrast STEM imaging with a high-angle annular dark field (HAADF) detector. The measured STEM intensity is quantified by comparing the atom column intensity with accurate image simulation from which we obtain the local composition. The measured morphology of the nanostructures then forms the basis for the interpretation of optical properties. The applied quantitative STEM method uses STEM intensity averaged within Voronoi cells around the atom column positions. Comparison with image simulation requires normalization of the STEM intensity with respect to the intensity of the incident electron beam, for which we use a detector scan [5] taking care of instrumental imperfections [6]. Simulated STEM intensity is obtained with the frozen lattice approach of the STEMsim program [7] taking the non-uniform detector sensitivity into account. In case of alloys containing atoms with different covalent radii (e.g. In and $\mathrm{Ga}$ in $\operatorname{In}_{\mathrm{x}} \mathrm{Ga}_{1-\mathrm{x}} \mathrm{N}$ ) static atomic displacements are computed with empirical potentials and included in the simulation, as they contribute to the STEM intensity by diffuse Huang scattering.

Figure 1 shows results obtained for Stranski-Krastanow-grown InGaAs QDs [1]. High-resolution STEM reveals an InGaAs wetting layer with a height of 8 mono layers having approximately lens-shaped QDs on top with heights of 2-4 nm and diameters between $5 \mathrm{~nm}$ and $20 \mathrm{~nm}$ (samples A). For samples B, an additional $4 \mathrm{~nm}$ thick strain reducing layer (SRL) containing nominally $10 \%$ indium was grown. Figure 1b shows a map of the measured In-concentration and Figs. 1c and d depict In-concentration profiles of wetting layer and quantum dot area, respectively. Figure 1a contains PL spectra in comparison with the position of emission lines obtained theoretically using combined tight-binding and configurationinteraction calculations directly based on the structural TEM data. Figure 2 shows results from an array of core-shell nanowires [2]. Figure 2a gives an overview of the CL intensity and Fig. 2b depicts the core-shell area. Figure 2c shows the CL intensity as a function of the wavelength, revealing a significant red shift from the bottom to the top of a nanowire. This can be understood by inspecting the maps of the In-concentration shown in Fig. 2e and Fig. 2g corresponding to the top and the bottom of a nanowire, respectively. The maps show that the thickness as well as the In-concentration of the InGaN QW increase along the nanowire, both leading to a red shift of the wavelength of the emitted light. 


\section{References:}

[1] E Goldmann et al, Applied Physics Letters 105 (2014), p. 152102.

[2] M Müller et al, Nano Letters 16 (2016), p. 5340.

[3] F F Krause et al, Ultramicroscopy 156 (2015), p. 29.

[4] K Müller-Caspary et al, Scientific Reports 6 (2016), p. 37146.

[5] T Mehrtens et al, Ultramicroscopy 131 (2013), p. 1.

[6] F F Krause et al, Ultramicroscopy 161 (2016), p. 146.

[7] A Rosenauer et al, Springer Proceedings in Physics 120 (2007), p. 169
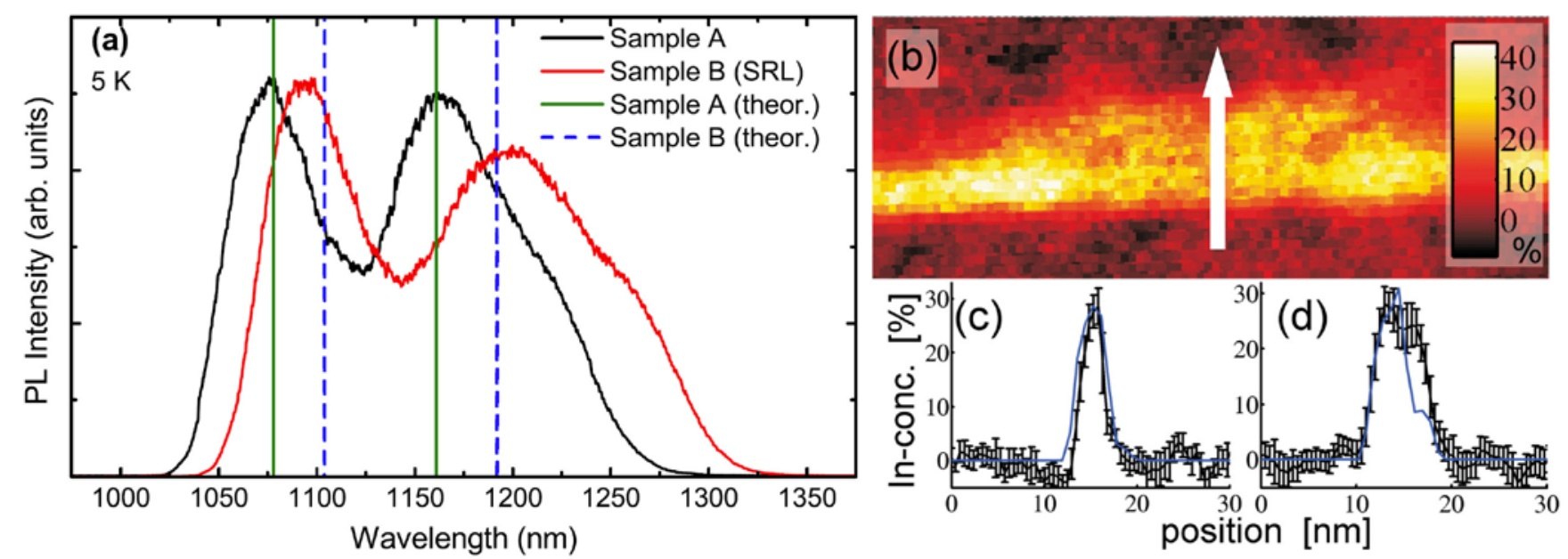

Figure 1. (a) Low temperature PL spectra of samples A and B at an excitation power of $30 \mathrm{~mW} / \mathrm{cm}^{2}$ exhibiting a red shift due to the SRL and emission energies calculated by empirical tight binding theory according to the structural data derived from high-resolution STEM. (b) map of the projected Inconcentration obtained from quantitative evaluation of the STEM intensity (sample A). (c) and (d) concentration profiles through the sole wetting-layer and the QD area, respectively.
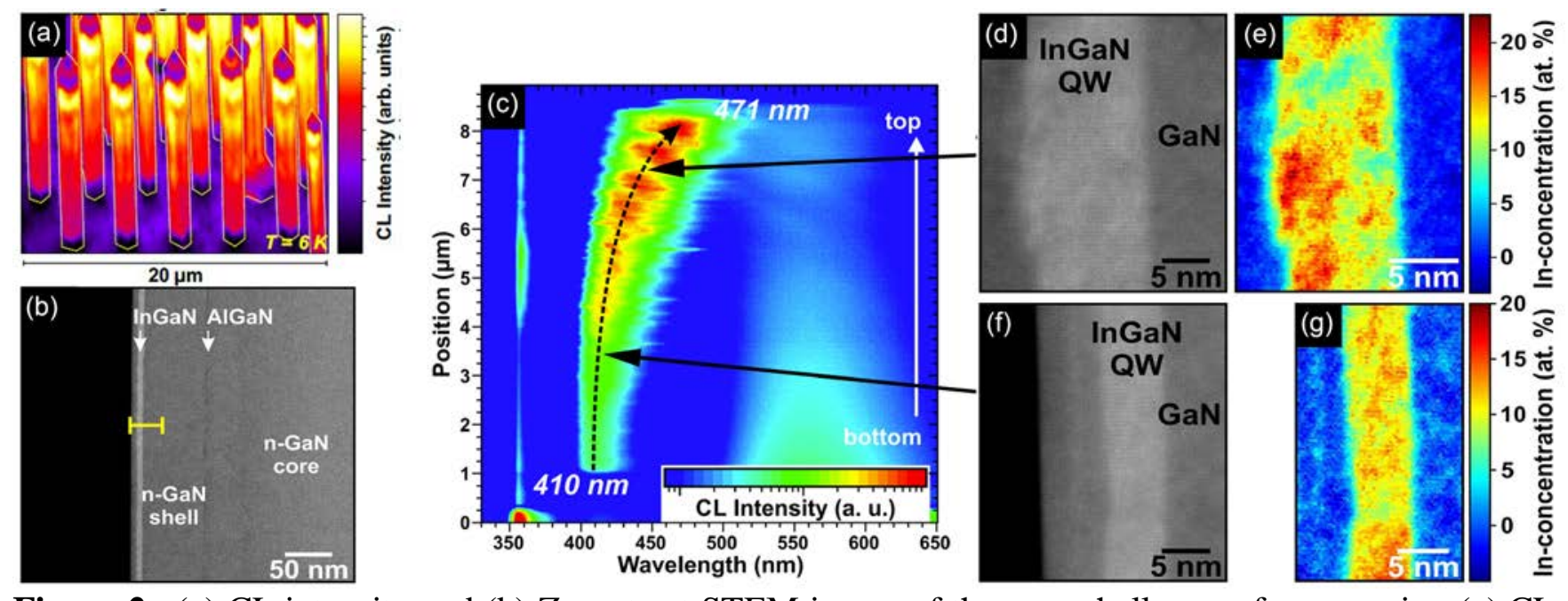

Figure 2. (a) CL intensity and (b) Z-contrast STEM image of the core-shell area of a nanowire. (c) CLintensity (color coded) plotted as a function of the wavelength from bottom to the top of a nanowire. (d) and (f) STEM images of the quantum well (QW) corresponding to the top and the bottom of a nanowire, respectively. The corresponding evaluated In-concentration maps are shown in (e) and (g). 